\title{
The rationale for routine cerebral ultrasound in premature infants
}

\author{
Maria I. Argyropoulou • Corinne Veyrac
}

Received: 4 October 2013 / Revised: 3 February 2014 / Accepted: 27 March 2014

(C) Springer-Verlag Berlin Heidelberg 2014

Premature birth is associated with brain lesions mostly resulting from hypoxia-ischaemia and haemorrhage $[1,2]$. Hypoxiaischaemia may give rise to periventricular leukomalacia (PVL) in either the focal form or the diffuse form [3]. Focal PVL is characterized by necrosis of all cell elements in the periventricular white matter, with the formation of cysts that progressively coalesce, resulting in ventricular enlargement with irregular outlines of the lateral ventricles and thinning of the periventricular white matter [3, 4]. Diffuse PVL is characterized by apoptotic cell death of the oligodendroglial precursors, resulting in hypomyelination and ventricular enlargement with regular outlines $[3,5]$. An increased incidence $(50 \%)$ of periventricular leukomalacia is reported in very-low-birthweight $(\leq 1,500 \mathrm{~g})$ premature infants, with the most severe cystic form representing only $5 \%[6]$.

Haemorrhagic lesions take the form of a continuum of germinal matrix haemorrhage, intraventricular haemorrhage and periventricular venous haemorrhagic infarction. The factors underlying the development of germinal matrix haemorrhage, which is apparently of venous origin, are increased vascularity, vascular immaturity and deficient autoregulation of cerebral blood flow [7]. Germinal matrix haemorrhage may progress to intraventricular haemorrhage, with or without ventricular dilatation. Periventricular haemorrhagic infarction is almost always associated with intraventricular haemorrhage, and compression of the paraventricular veins belonging to the deep Galenic system of drainage is thought to be the underlying cause $[1,2]$.

M. I. Argyropoulou ( $\square)$

Department of Radiology, University of Ioannina Medical School,

45110 Ioannina, Greece

e-mail: margyrop@cc.uoi.gr

C. Veyrac

Department of Paediatric Radiology, Arnaud de Villeneuve Hospital, Montpellier, France
Germinal matrix haemorrhage with intraventricular haemorrhage occurs in $20 \%$ of very-low-birth-weight premature infants, while intraventricular haemorrhage associated with periventricular haemorrhagic infarction occurs in less than $5 \%$ of cases [6]. An increased incidence of periventricular haemorrhagic infarction is observed in infants with birth weight less than $750 \mathrm{~g}$ [6].

Post-haemorrhagic hydrocephalus, which can be communicating or non-communicating, is another complication of intraventricular haemorrhage $[1,2]$. The incidence of posthaemorrhagic ventricular dilation is $36 \%$, and this resolves in approximately $65 \%$ of cases [1]. Cerebellar abnormalities, mainly haemorrhage, infarction and underdevelopment, are being increasingly recognized and these have been associated with very low birth weight $[4,8]$. Hemispheric haemorrhage, which is the most common cerebellar haemorrhagic lesion, is usually unilateral, starting in the external granular layer [8]. The less common vermian haemorrhage starts in the germinal matrix of the fourth ventricle [8]. The incidence of cerebellar haemorrhage is $17 \%$ in infants weighing less than $750 \mathrm{~g}$ and $2 \%$ in infants more than $750 \mathrm{~g}$ at birth [8]. Cerebellar infarction has been associated with supratentorial periventricular leukomalacia, and its reported incidence in an unselected population is $10-15 \%$ [9]. Underdevelopment is the most common cerebellar abnormality, for which the predisposing factors are extreme prematurity, very low birth weight, haemosiderin blood products, severe hypotension, large patent ductus arteriosus and transynaptic trophic effects [3].

Other acquired lesions of the premature brain are punctate white matter hyperintensities, perforator stroke and infectious lesions [10-12]. Punctate white matter hyperintensities are relatively common lesions (incidence $20 \%$ ), occurring in the preterm period and decreasing in number at term-equivalent age [10]. Perforator stroke occurs in the distribution of lenticulostriate arteries, and in preterm neonates it is often subclinical [12]. Viral or fungal infections can affect the brain 
of very-low-birth-weight infants and these can give rise to multicystic periventricular leukomalacia, multiple brain abscesses and haemorrhagic infarcts [13-16].

Early detection and follow-up of brain lesions in premature infants is important so appropriate treatment can be given and prognosis can be improved. Various imaging techniques, mainly US and MRI, have been used for this purpose [1, 2]. US was the first imaging modality to be used, in the 1980s. Even in the era of diffusion tensor imaging (DTI), functional MRI and voxel-wise analysis of MRI data [17], US remains the first and, until the premature baby is clinically stable, the only imaging modality widely available for the evaluation of the infant brain. Is this good enough for the future of premature babies? Or do we need desperately to invest in MRcompatible incubators to image these fragile infants safely? Is the practice of US appropriate, adequate and safe in this situation? To evaluate the rationale for routine cerebral US in premature infants we should be able to answer the following three questions: (1) Why do we perform US?, (2) How do we perform US? and (3) When do we perform US?

\section{Why do we perform US?}

Cerebral US is a non-invasive, radiation-free imaging technique that can be performed without sedation at the bedside of intubated, ventilated infants who would be difficult to examine using MRI outside an MR-compatible incubator [1]. But is US accurate enough when compared with the gold standard for brain imaging, which is MRI? Close correlation between brain US and MRI findings has been reported, provided that meticulous US technique is applied [18].

\section{How do we perform US?}

The brain US examination consists of grey-scale imaging of the brain parenchyma and colour/pulsed Doppler of the vessels [1]. Sectorial with small footprint (5- to 8-MHz) and linear-array (5- to $12-\mathrm{MHz}$ ) transducers should be used. The main acoustic window is the anterior fontanelle but the mastoid and posterior fontanelles are also used in most cases. We propose systematic application of a standard protocol. The grey-scale imaging begins with the sectorial transducer, using the acoustic window of the anterior fontanelle, and comprises a series of five to seven coronal scans and five sagittal scans [19] (Tables 1 and 2). A midline sagittal and a coronal scan performed with the linear-array transducer are also useful. With this series of slices the ventricles (shape, size and content), the white matter (periventricular and subcortical), the thalami and basal ganglia, the grey matter and the pericerebral spaces can be assessed accurately.
Table 1 US imaging of the infant brain using the acoustic window of the anterior fontanelle: coronal slices on grey-scale imaging

Coronal

Slice 1

Interhemispheric fissure

Frontal lobes

Slice 2

Interhemispheric fissure

Frontal horns

Slice 3

Interhemispheric fissure

Frontal horns

Sylvian fissures

Caudate nucleus

Putamen

Globus pallidus

Temporal lobes

Slice 4

Interhemispheric fissure

Lateral ventricles

Third ventricle

Thalamus

Cerebral peduncles

Cerebellum

Slice 5

Interhemispheric fissure

Lateral ventricle

Third ventricle

Thalamus

Quadrigeminal plate

Hippocampus

Cerebellum

Fourth ventricle

Slice 6

Interhemispheric fissure

Lateral ventricle, glomus of choroid plexus

Tentorium

Slice 7

Interhemispheric fissure

Parietal and occipital lobes

The mastoid fontanelle, the "star fontanelle" or asterion (the Greek word for star), should always be used for detailed evaluation of the posterior fossa. At least two slices should be performed, depicting the cerebral peduncles, the vermis, the cerebellar hemispheres, the fourth ventricle, the quadrigeminal plate and the cisterna magna [20]. The posterior fontanelle may be used to better visualize the occipital lobes and occipital horns and to distinguish between a prominent choroid plexus and a small intraventricular haemorrhage [1]. 
Table 2 US imaging of the infant brain using the acoustic window of the anterior fontanelle: sagittal slices on grey-scale imaging

\begin{tabular}{|c|c|}
\hline Sagittal & \\
\hline Slice 1 & $\begin{array}{l}\text { Midline } \\
\text { Cingulate gyrus } \\
\text { Corpus callosum } \\
\text { Third ventricle, choroid plexus } \\
\text { Brainstem } \\
\text { Vermis } \\
\text { Fourth ventricle }\end{array}$ \\
\hline Slice 2 & $\begin{array}{l}\text { Left parasagittal } \\
\text { Lateral ventricles, choroid plexus } \\
\text { Caudate nucleus head } \\
\text { Thalamus } \\
\text { Caudothalamic groove } \\
\text { Cerebellar hemisphere }\end{array}$ \\
\hline Slice 3 & $\begin{array}{l}\text { Right parasagittal } \\
\text { Lateral ventricles, choroid plexus } \\
\text { Caudate nucleus head } \\
\text { Thalamus } \\
\text { Caudothalamic groove } \\
\text { Cerebellar hemisphere }\end{array}$ \\
\hline Slice 4 & $\begin{array}{l}\text { Left peripheral parasagittal } \\
\text { Frontal lobe } \\
\text { Parietal lobe } \\
\text { Temporal lobe } \\
\text { Sylvian fissure }\end{array}$ \\
\hline Slice 5 & $\begin{array}{l}\text { Right peripheral parasagittal } \\
\text { Frontal lobe } \\
\text { Parietal lobe } \\
\text { Temporal lobe } \\
\text { Sylvian fissure }\end{array}$ \\
\hline
\end{tabular}

Pulsed Doppler provides evaluation of spectra of the anterior and middle cerebral arteries. Depending on the possible pathological findings detected on grey-scale imaging (e.g., hydrocephalus), compression at the anterior fontanelle may be applied. On colour Doppler, evaluation for alternating coloured echoes into the aqueduct of Sylvius is useful to distinguish between a large subependymal haemorrhage and an intraventricular haemorrhage. Evaluation for the presence of flow in the terminal veins is performed in very-low-birthweight premature babies with intraventricular haemorrhage because absence of flow is highly indicative of a pending venous infarct [1].

\section{When do we perform US?}

Grey-scale imaging with colour Doppler should be performed in all neonates born at less than 32 weeks of gestation, initially within $72 \mathrm{~h}$ of birth, then at least weekly until discharge from the hospital, and again at about term-equivalent age [11]. Studies using this protocol [21, 22] have demonstrated a higher sensitivity in predicting outcomes than those applying brain US on a less frequent basis [23, 24]. Serial US will not miss: (1) periventricular cysts of focal periventricular leukomalacia (PVL), which appear after the first postnatal week, progressively coalesce and then disappear at about term-equivalent age by forming the wall of the lateral ventricles $[4,11]$; (2) periventricular clastic lesions secondary to sepsis, necrotizing enterocolitis and viral and fungal infections developing any time in the neonatal period [11, 14-16, 25]; (3) post-haemorrhagic ventricular dilatation that may develop any time after intraventricular hemorrhage [26], and (4) perforator stroke occurring any time after the first week and sometimes affecting the posterior limb of the internal capsule [12].

\section{A useful check-list for the first US}

One should look for:

(1) lesions of antenatal origin (e.g., congenital malformations and clastic lesions).

(2) germinal matrix or intraventricular haemorrhage; 70$90 \%$ of haemorrhages appear in the first $72 \mathrm{~h}$ after birth, with the incidence increasing with decreasing gestational age and birth weight, and early haemorrhage carries a higher risk of worsening [1,27].

(3) an abnormal arterial Doppler spectrum (fluctuating pattern, extremely high velocities or very low velocities) that can be considered predictive of the development of intraventricular haemorrhage [28].

(4) a strongly hyperechogenic focal lesion that suggests intraparenchymal haemorrhage.

(5) an abnormal parenchymal echogenicity around the lateral ventricles, appearing as hyperechogenicity with spiculated or sharp margins, or as punctate or focal nodular lesions. These abnormal patterns, which suggest pending focal periventricular leukomalacia, should be differentiated from the periventricular echogenic stripes parallel to the ventricular wall seen in extremely premature infants and from transient increased periventricular hyperechogenicity that may be observed during the first days of life [1].

(6) a cerebellar lesion - infarct, haemorrhage or underdevelopment [8]. 
All these anomalies should be described meticulously and located accurately.

\section{When the first US shows abnormalities, how do we proceed?}

(1) If the only lesion is a hyperechogenic germinal matrix haemorrhage developing into a cyst, this can be expected to disappear within 10 days to 3 months, and no follow-up is necessary beyond the 1st month of life [1].

(2) If the lesion is a small intraventricular haemorrhage, the hyperechogenic clot can be expected to disappear within 6 weeks [1].

(3) If the lesion is a large intraventricular haemorrhage, this carries a high risk of development of post-haemorrhagic ventricular dilatation and requires US follow-up every 37 days. If the lesion is a periventricular haemorrhagic infarction, repeated US will monitor the development of cysts. Periventricular venous haemorrhagic infarctions extending into the para-atrial area carry a high risk of development of hemiplegic cerebral palsy [29]. Posthaemorrhagic hydrocephalus can develop into a communicating form that carries a better prognosis and should be differentiated from the non-communicating form of hydrocephalus with marked enlargement of the 4th ventricle, which necessitates invasive treatment. Pulsed Doppler with and without manual compression of the anterior fontanelle is useful for evaluation of progression of the hydrocephalus or assessment of the efficacy of lumbar or ventricular puncture or shunting [1]. The Delta RI index, derived as: fontanelle compression (RI-baseline RI)/baseline RI is useful for identifying subjects requiring shunt placement (i.e., with Delta RI $>45 \%$ ) [1].

(4) If the initial lesion consists of abnormal echogenicity around the occipital horns of the lateral ventricles, the weekly US follow-up should depict either cystic periventricular leukomalacia or a normal appearance [2].

(5) Brain US performed at term-equivalence is useful to detect ventricular enlargement, a hypoplastic corpus callosum and enlarged pericerebral spaces.

\section{When do we proceed to MRI?}

A baseline MRI should be performed at about term-equivalent age in premature infants with US findings of focal periventricular leukomalacia, periventricular haemorrhagic infarction, persistent post-haemorrhagic hydrocephalus, perforator stroke and infectious lesions.

\section{But what is the added value of MRI at this stage?}

Does MRI information affect the clinical management and thus the long-term prognosis of the individual patient or does it just provide data for studies that may offer a better understanding of diseases and probably affect clinical practices in the future? The answer is both. Protocols for brain MRI include, among others, a 3-D T1-weighted sequence and diffusion tensor imaging (DTI) [30]. For the individual patient T1-weighted images offer invaluable information about the myelination status of the internal capsule: A normal appearance at about term-equivalent age is a symmetrical high signal intensity of the posterior limb of the internal capsule. Lack of high signal intensity, asymmetry and even small lesions have been associated with abnormal motor outcomes, ranging from motor asymmetry to cerebral palsy $[11,31]$. DTI offers additional information on white matter maturation and integrity [31]. Finally, by using standard protocols and image parameters, data from 3-D T1-weighted sequences and DTI can be analysed with voxel-wise techniques to extract useful information from a large series of patients [17]. This information might offer deeper pathophysiological insight that is likely to dictate better clinical practice in the future.

Conflicts of interest None

\section{References}

1. Veyrac C, Couture A, Saguintaah M et al (2006) Brain ultrasonography in the premature infant. Pediatr Radiol 36:626-635

2. Argyropoulou MI (2010) Brain lesions in preterm infants: initial diagnosis and follow-up. Pediatr Radiol 40:811-818

3. Volpe JJ (2009) The encephalopathy of prematurity — brain injury and impaired brain development inextricably intertwined. Semin Pediatr Neurol 16:167-178

4. Argyropoulou MI, Xydis V, Drougia A et al (2003) MRI measurements of the pons and cerebellum in children born preterm; associations with the severity of periventricular leukomalacia and perinatal risk factors. Neuroradiology 45:730-734

5. Tzarouchi LC, Xydis V, Zikou AK et al (2011) Diffuse periventricular leukomalacia in preterm children: assessment of grey matter changes by MRI. Pediatr Radiol 41:1545-1551

6. Volpe JJ (2009) Brain injury in premature infants: a complex amalgam of destructive and developmental disturbances. Lancet Neurol 8 : $110-124$

7. Ballabh $P$ (2010) Intraventricular hemorrhage in premature infants: mechanism of disease. Pediatr Res 67:1-8

8. Volpe JJ (2009) Cerebellum of the premature infant: rapidly developing, vulnerable, clinically important. J Child Neurol 24:1085-1104

9. Mercuri E, He J, Curati WL et al (1997) Cerebellar infarction and atrophy in infants and children with a history of premature birth. Pediatr Radiol 27:139-143

10. Bassi L, Chew A, Merchant N et al (2011) Diffusion tensor imaging in preterm infants with punctate white matter lesions. Pediatr Res 69: $561-566$ 
11. De Vries LS, Benders MJ, Groenendaal F (2013) Imaging the premature brain: ultrasound or MRI? Neuroradiology 55:13-22

12. Abels L, Lequin M, Govaert P (2006) Sonographic templates of newborn perforator stroke. Pediatr Radiol 36:663-669

13. Verboon-Maciolek MA, Truttmann AC, Groenendaal F et al (2012) Development of cystic periventricular leukomalacia in newborn infants after rotavirus infection. J Pediatr 160:e161

14. Verboon-Maciolek MA, Groenendaal F, Hahn CD et al (2008) Human parechovirus causes encephalitis with white matter injury in neonates. Ann Neurol 64:266-273

15. Marcinkowski M, Bauer K, Stoltenburg-Didinger G et al (2001) Fungal brain abscesses in neonates: sonographic appearances and corresponding histopathologic findings. J Clin Ultrasound 29:417-421

16. Pahud BA, Greenhow TL, Piecuch et al (2009) Preterm neonates with candidal brain microabscesses: a case series. J Perinatol 29:323-326

17. Astrakas LG, Argyropoulou MI (2010) Shifting from region of interest (ROI) to voxel-based analysis in human brain mapping. Pediatr Radiol 40:1857-1867

18. Epelman M, Daneman A, Kellenberger CJ et al (2010) Neonatal encephalopathy: a prospective comparison of head US and MRI. Pediatr Radiol 40:1640-1650

19. Lowe LH, Bailey Z (2011) State-of-the-art cranial sonography: part 2, pitfalls and variants. AJR Am J Roentgenol 196:1034-1039

20. Enriquez G, Correa F, Aso C et al (2006) Mastoid fontanelle approach for sonographic imaging of the neonatal brain. Pediatr Radiol 36:532-540

21. De Vries LS, Van Haastert IL, Rademaker KJ et al (2004) Ultrasound abnormalities preceding cerebral palsy in high-risk preterm infants. $\mathrm{J}$ Pediatr 144:815-820
22. Leijser LM, Liauw L, Veen S et al (2008) Comparing brain white matter on sequential cranial ultrasound and MRI in very preterm infants. Neuroradiology 50:799-811

23. Mirmiran M, Barnes PD, Keller K et al (2004) Neonatal brain magnetic resonance imaging before discharge is better than serial cranial ultrasound in predicting cerebral palsy in very low birth weight preterm infants. Pediatrics 114:992-998

24. Woodward LJ, Anderson PJ, Austin NC et al (2006) Neonatal MRI to predict neurodevelopmental outcomes in preterm infants. N Engl $\mathrm{J}$ Med 355:685-694

25. Leijser LM, de Vries LS, Cowan FM (2006) Using cerebral ultrasound effectively in the newborn infant. Early Hum Dev 82:827-835

26. Van Wezel-Meijler G, Steggerda SJ, Leijser LM (2010) Cranial ultrasonography in neonates: role and limitations. Semin Perinatol 34:28-38

27. Van de Bor M, Van Bel F, Lineman R et al (1986) Perinatal factors and periventricular-intraventricular hemorrhage in preterm infants. Am J Dis Child 140:1125-1130

28. Perlman JM, Goodman S, Kreusser KL et al (1985) Reduction in intraventricular hemorrhage by elimination of fluctuating cerebral blood-flow velocity in preterm infants with respiratory distress syndrome. N Engl J Med 312:1353-1357

29. Lahutte M, Bordarier C, Hornoy P et al (2010) Venous infarction of the neonate. J Radiol 91:787-796

30. Tzarouchi LC, Drougia A, Zikou A et al (2014) Body growth and brain development in premature babies: an MRI study. Pediatr Radiol 44:297-304

31. Cowan FM, de Vries LS (2005) The internal capsule in neonatal imaging. Semin Fetal Neonatal Med 10:461-474 